\title{
PAIR-BREAKING MECHANISMS IN Nb/Gd/Nb FILMS
}

\author{
C. Strunk, U. Paschen, C. Sürgers, H. v. Löhneysen
}

Physikalisches Institut, Universität Karlsruhe, 7500 Karlsruhe, Germany

Several series of $\mathrm{Nb} / \mathrm{Gd} / \mathrm{Nb}$ triple layers have been grown in UHV by $\mathrm{e}^{-}$-beam evaporation onto sapphire $(11 \overline{2} 0)$ substrates at $300 \mathrm{~K}$. The superconducting transitions have been measured resistively and by dc-susceptibility, the ferromagnetic transition with the transverse magnetooptical Kerr effect. The Gd layers show long-range ferromagnetic order only above a layer thickness of $20 \AA$, which is attributed to the formation of a discontinuous film below this thickness. The superconducting properties of the films reflect this transition by a sudden decrease in the $T_{c}\left(d_{G d}\right)$-curve with increasing $d_{G d}$. This result indicates that pair breaking by the bulk-Gd exchange field is much more effective than pair breaking by spin-flip scattering. Depending on the thickness of the $\mathrm{Nb}$ layers, $T_{c}\left(d_{G d}\right)$ saturates for large values of $d_{G d}$ at a finite temperature or tends to zero.

In classic investigations of the proximity effect between superconducting (SC) and ferromagnetic (FM) films the observed pair-breaking effects have been explained by a combination of the usual DeGennes-Werthamer (DGWH) theory with the Abrikosov-Gorkov (AG) model of dilute, paramagnetic spin impurities [1]. Though fair agreement between theory and experiment has been achieved, the assumption of independent spins in a concentrated FM remained hard to justify. Recently, the penetration of Cooper pairs into a FM has be described theoretically and yields good agreement between theory and experiments [2]. The samples investigated in this work show a transition from paramagnetic (PM) to FM behavior with increasing thickness of Gd layers $d_{G d}$ and are thus well suited to compare the influence on superconductivity of both - random and FM ordered spins - with the corresponding theories.

The samples were grown by $\mathrm{e}^{-}$-beam evaporation at room temperature as described in [3]. We prepared eight series of $\mathrm{Nb} / \mathrm{Gd} / \mathrm{Nb}$ layers, keeping the $\mathrm{Nb}$ thickness $d_{N b}$ constant within each series. $d_{N b}$ was chosen equal in both $\mathrm{Nb}$ films with exception of the $168 \AA$ series. During the evaporation, $d_{G d}$ was varied by successively covering 18 substrates with a moving shutter. In this way, we got constant material parameters of the $\mathrm{Nb}$ films within a series, which leads to a low scatter in the resulting SC transition temperatures $T_{c}\left(d_{G d}\right)$. The growth of the Gd films has been monitored during the evaporation by RHEED. The peak of the RHEED reflections showed at $d_{G d} \approx 10 \AA$ a shift from the $\mathrm{Nb}$ to the $\mathrm{Gd}$ position, while it reached the original sharpness not before $d_{G d} \approx 20 \AA$. From this, we conclude that the Gd layers grow at first in an island mode before a continuous film is formed above $d_{G d} \approx 20 \AA$. Only the continuous films exhibit FM order as demonstrated by measuring the magnetooptical Kerr effect for samples with $d_{G d}=15,20$ and $25 \AA$. The latter two show a FM magnetization below $T_{\text {Curie }} \approx 140 \mathrm{~K}$ and $225 \mathrm{~K}$ respectively. The $\mathrm{SC}$ transition temperatures, determined resistively by the midpoint of the transition, show a continuous depression with increasing $d_{G d}$ (Fig. 1). For the series with $d_{N b}=$ $191 \AA, T_{c}$ has been checked additionally by SQUID magnetometry.

At high $d_{G d}, T_{c}$ saturates or tends to zero, depending on $d_{N b}$. Around $d_{G d} \approx 20 \AA$, a sudden drop appears in the curves. As suggested by the structural and magnetic characterization of the Gd layers, we attribute this structure to the onset of long range magnetic order. In the PM range $\left(d_{G d} \leq 20 \AA\right)$ spin-flip scattering should give the dominant pairbreaking effect at the boundary. With the evolution of FM order, the spin-flip scattering rate should be reduced by several orders of magnitude, because single Gd spins cannot be flipped against the exchange field of their surrounding. Therefore in the FM range another microscopic mechanism must be present. Radovic et al. [2] have explained the pair breaking inside a ferromagnet by the exchange shift of the Gd conduction (sub-)bands $I$, which makes the usual $(\vec{k},-\vec{k})$ pairing unfavorable. Consequently, the $T_{c}$ reduction in the PM and FM range are interpreted in terms of the two theories $\{1,2]$ (Fig.2). The data points are taken from the plateau values at $d_{G d} \approx 13 \AA(\mathrm{PM})$ and $d_{G d} \approx 30 \AA(\mathrm{FM})$ 


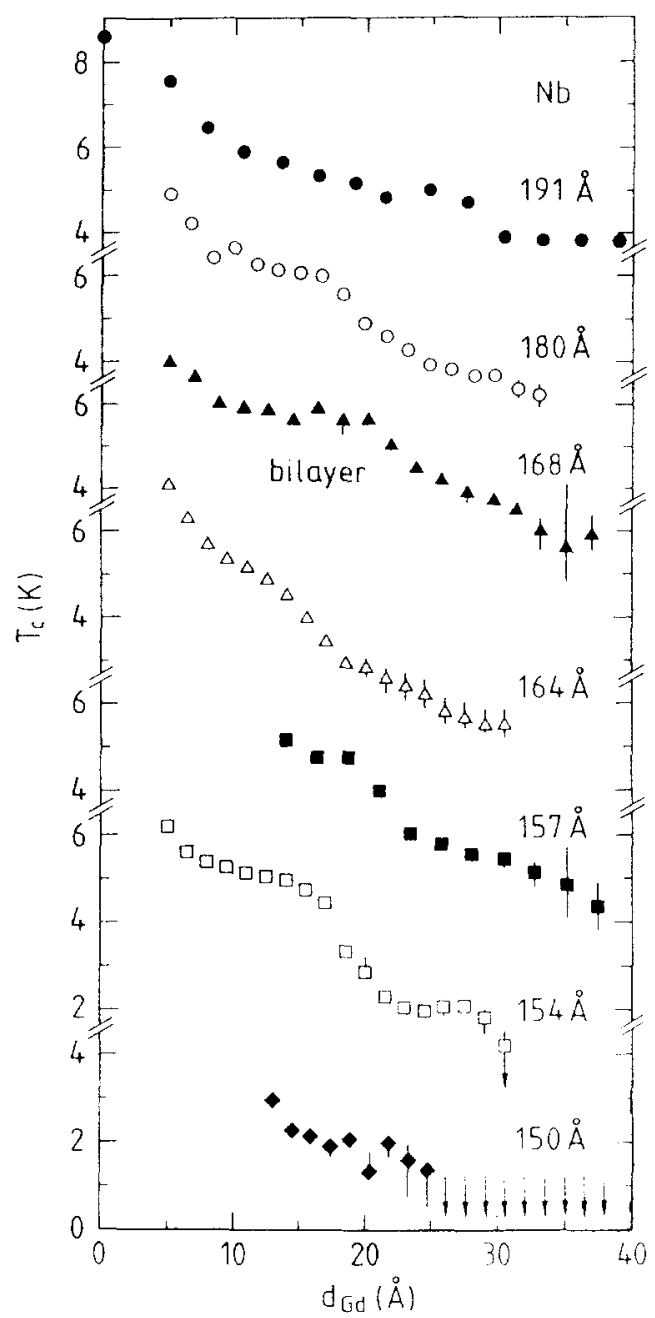

Fig. 1: $T_{c}\left(d_{G d}\right)$ for different values of $d_{N b}$

The depairing by the exchange splitting turns out to be much more effective than that by spin-flip scattering.

Three parameters enter both theories : the SC coherence length $\xi_{S}$, the penetration depth $\left(k_{M}\right)^{-1}$, and a coefficient $\eta$, stemming from the (generalized) DGWH boundary condition [2]. $\quad \xi_{S}=78 \AA$ and $\eta=0.045$ have been determined from $T_{c}\left(d_{N b}\right)$ at $d_{G d} \approx 34 \AA$ [4]. Fitting both theories to the data yields values for $\left(k_{M}\right)^{-1}$, the effective spin-flip scattering time $\tau_{S}$ and $I$ (estimating the mean free path $\ell_{G d} \approx d_{G d}$ ) making use of $k_{M}=1 / v_{F} \tau_{S}$ (PM) and $k_{M}=(1+\mathrm{i}) \cdot 2 / \xi_{M} \xi_{M}=\left(4 \hbar D_{M} d\right)^{1 / 2}, D_{M}=v_{F} \ell_{G d} / 3$ (FM) (Table 1).

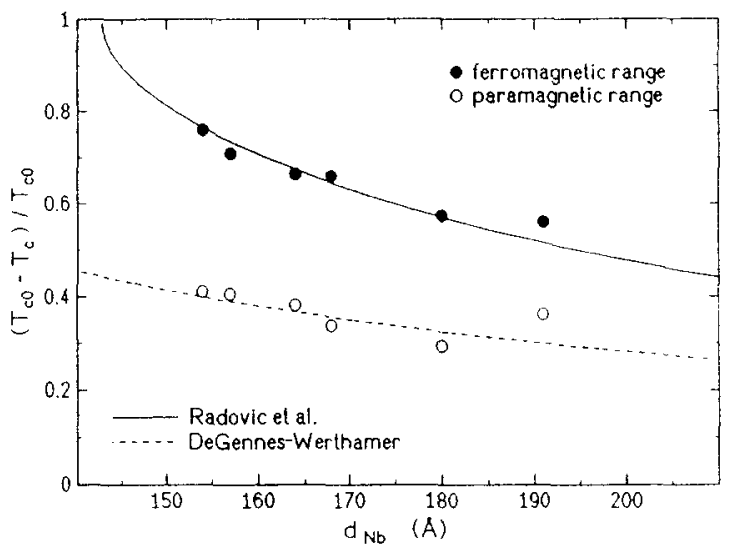

Fig. 2: $T_{c}$ reduction in the FM and $\mathrm{PM}$ ranges

The data in the FM range can also be fitted by the DGWH theory but this requires a decrease of the effective spin-flip scattering time with the evolution of the magnetic order. Since $\tau_{S}$ should increase with increasing exchange field this interpretation seems not appropriate. Fitting Radovic's theory to the data gives for the exchange shift a value $I$ considerably lower than the bulk value $I_{o}=0.31 \mathrm{eV} \mathrm{[5]}$. This is in qualitative accord with the decrease of $T_{\text {Curie }}$ at low $d_{G d}$. The fact that $k_{M}$ is complex gives rise to spatial oscillations of the $\mathrm{SC}$ order parameter, which should show up in $T_{c}\left(d_{G d}\right)$ also [6]. To observe this effect, the samples have to be ferromagnetic down to $d_{F M} \approx \xi_{M} / 2 \approx 5-7 \AA$.

\begin{tabular}{|c|c|c|c|c|c|}
\hline range & theory & $\left(k_{\Lambda}\right)^{-1}(\AA)$ & $\tau_{S}(\mathrm{sec})$ & $\xi_{\Delta}(\AA)$ & $I(\mathrm{eV})$ \\
\hline PM & DGWH & 9.2 & $7.9 \cdot 10^{-15}$ & - & - \\
\hline FM & Radovic & - & - & 13.4 & 0.17 \\
\hline FM & DGWH & 4.5 & $3.8 \cdot 10^{-15}$ & - & - \\
\hline
\end{tabular}

Table 1 : Parameters determined from fits in Fig. 2

In conclusion, we have observed that the onset of FM order in our films is associated with a change in the underlying pair-breaking mechanism.

\section{REFERENCES}

1. J. J. Hauser et al., Phys. Rev. 142, 118 (1966)

2. Z. Radovic et al., Phys. Rev. B38, 2388 (1988)

3. C. Sürgers, H. v. Löhneysen, Thin Solid Films 219, 69 (1992)

4. C. Strunk. et al., to be published at this Conference

5. A. J. Freeman, in : R. J. Elliott (Ed.), Magnetic Properties of Rare Earth Metals (Plenum Press, London 1972), p. 311

6. Z. Radovic et al., Phys. Rev. B44, 759 (1991) 